\title{
LA VIDA EN LAS AULAS: IMPLICACIONES DE “APRENDER A APRENDER" EN LA EDUCACIÓN SECUNDARIA Y SUPERIOR
}

\section{CLASSROOM LIFE: IMPLICATIONS OF “LEARNING TO LEARN" IN SECONDARY AND HIGHER EDUCATION}

\author{
Florentino Silva Becerra \\ cienaga16m@hotmail.com \\ Martha Valadez Huizar \\ martha_vala@hotmail.com
}

\section{Centro Universitario de Ciencias Sociales y Humanidades de la \\ Universidad de Guadalajara, MÉXico}


Para citar este artículo:

Silva Becerra, F., \& Valadez Huizar, M. (2020). La vida en las aulas: implicaciones de "aprender a aprender" en la educación Secundaria y Superior. ESPACIO I+D, INNOVACIÓN MÁS DESARROLLO, 9(24). https://doi.org/10.31644/IMASD.24.2020.a06

\section{RESUMEN}

La transformación de la docencia versus la transformación del conocimiento, donde las circunstancias propias de los cambios de la naturaleza humana se obstruyen ante el cambio paralelo, se impone la innovación, mediante la participación creativa para la incorporación a la vida del siglo XXI. El gran desafío consiste en la transformación de nuestro pensamiento, que se aferra al marco tradicional, donde el reaprender y desaprender de forma permanente implican procesos de la complejidad educativa actual. Ante este escenario, las instituciones educativas se encuentran en la intrincada situación de realizar cambios en su estructura. ¿Cuál es la discrepancia entre el marco institucional y el marco de la sociedad del conocimiento? ¿Cuáles son las situaciones que se involucran en el proceso de enseñanza y aprendizaje de los alumnos y profesores? Su objetivo es demostrar la distancia entre el postulado educativo actual -aprender a aprender- y las situaciones que se viven en el proceso de enseñanza y aprendizaje en la educación secundaria y superior. El proceso de autorregulación para aprender a aprender, representa un concepto deseable más no sostenido por la práctica educativa. Si bien lo que prevalece son las prácticas tradicionales y los entornos educativos excluyentes que niegan el acceso al cambio educativo de la enseñanza y el aprendizaje, solo la vivencia metodológica permitirá comprender el proceso de aprender a aprender.

\section{Palabras clave}

Aprender a aprender, autorregulación, autonomía, globalización, complejidad y conocimiento. 
The transformation of teaching versus thetransformation of knowledge where the circumstances of the changes in human nature are obstructed by the parallel change, in order to respond to this situation, innovation is imposed through creative participation for incorporation into the life of the 21st century The great challenge will consist in the transformation of our thinking, which clings to the traditional framework, where relearning and unlearning permanently implies processes of current educational complexity. Faced with this scenario, educational institutions are in the intricate situation of making changes in their structure. What is the discrepancy between the institutional framework and the knowledge society framework? What are the situations that are involved in the teaching and learning process of students and teachers? Its objective is to show the distance between the current educational postulate -learning to learn- and the situations that are lived in the teaching and learning process in secondary and higher education. The process of self-regulation to learn to learn represents a desirable concept but not sustained by educational practice. Although what prevails are traditional practices and exclusive educational environments that deny access to educational change in teaching and learning, only the methodological experience will allow us to understand the process of learning to learn.

\section{Keywords}

Learning to learn, self-regulation, autonomy, globalization, complexity and knowledge. 
$\mathrm{E}$ 1 informe de la "Comisión Internacional para la Educación para el Siglo XXI”, asistida por la Organización de las Naciones Unidas para la Educación, la Ciencia y la Cultura (UNESCO) coordinado, por Jaques Delors, habla de la transformación de la docencia frente al fenómeno más trascendente de todas estas evoluciones: la revolución del conocimiento, como se llama a este espacio que actualmente vivimos. Al respecto, Brunner y Tedesco (2003, p. 23) manifiestan que: "el conocimiento prorrogó 1.750 años en duplicarse por primera vez, contando desde sus inicios de la era cristiana; luego duplicó su volumen cada 150 años, después cada 50 años. Ahora lo hace después de 5 años y se estima que para el año 2020 se duplicará cada 73 días". Como puede observarse, el conocimiento se desplaza a una velocidad incontenible, como consecuencia de este fenómeno, la enseñanza y el aprendizaje se someten a un ritmo de transformación que, por circunstancias propias de los cambios de la naturaleza humana, es casi imposible generar un estado paralelo, por lo que vivimos un mundo desafiante que, debido a las transformaciones inéditas, generadas por la globalización, vierte un mundo complejo.

Dice Delors (1996) sobre el pensar y edificar nuestro futuro común, que la educación tiene la misión de permitir a todos, sin excepción, hacer fructificar todos sus talentos y todas sus capacidades de creación, lo que implica que cada uno pueda responsabilizarse de sí mismo y realizar su proyecto personal.

La globalización es un fenómeno mundial que ha generado la revolución del conocimiento, los efectos en nuestro vivir responden a la innovación mediante la participación creativa para la incorporación a la vida del siglo XXI. El gran desafío consistiría en la transformación de nuestro pensamiento, que se aferra al marco tradicional, negando la posibilidad de generar una evolución que no va a la par con la creciente rapidez de los cambios y lo imprevisible, que caracteriza a este mundo (Mayor, 1999).

Al respecto, Morín (2000) propone la interdisciplinariedad para transformar nuestras formas de pensamiento, que implican reaprender $y$ desaprender de forma permanente, o lo que conceptualmente conocemos como aprender a aprender. Visión educativa que conduce a la modificación de nuestras prácticas pedagógicas, porque es en el aula donde se manifiestan nuestros comportamientos y se evidencian las formas de responder ante la complejidad educativa actual (Escotet, 1992).

Ante este escenario, las instituciones educativas se encuentran en la intrincada situación de realizar cambios en su estructura, difíciles de realizar porque implica la transformación de las formas de hacer gestión administrativa y académica. En este trabajo se pretende reflexionar sobre la discrepancia entre lo que se pide a las instituciones educativas, en el marco 
de la sociedad del conocimiento, y algunas situaciones que viven profesores y alumnos en la cotidianeidad de la impartición de una clase.

El propósito de este trabajo es mostrar la distancia entre el postulado educativo actual, aprender a aprender, que implica el desarrollo de procesos cognitivos complejos, y las situaciones que se viven en el proceso de enseñanza y aprendizaje en la educación básica y superior. Para el primer nivel, se toma la experiencia del director de una escuela secundaria; uno de los autores de este trabajo, para exponer que las dificultades de la vida cotidiana en un aula de clases, como el entorno de violencia, problemas de aprendizaje, emocionales, etc., limitan al profesor para lograr dicho propósito educativo, dado que tampoco cuenta con las herramientas necesarias para enfrentar estas situaciones.

Con este mismo enfoque, tratamos el tema en la educación superior, partiendo de la existencia de esta distancia entre la visión educativa del aprender a aprender, pero aquí con las prácticas pedagógicas, por lo que se exponen algunas estrategias de aprendizaje que pueden abonar a desarrollar procesos cognitivos que apoyen a la autogestión del estudiante.

\section{PERSPECTIVA TEÓRICA}

Hoy en día, en el campo de la educación se habla de autorregulación, autonomía y aprender a aprender, como procesos que permiten seguir aprendiendo a lo largo de la vida. Son conceptos que representan la dotación de herramientas intelectuales y sociales para acceder a un aprendizaje continuo, y como consecuencia, a una educación permanente que se genera como estrategia para hacer frente a las necesidades de aprendizaje del siglo XXI. Pero ¿Qué entendemos por estos conceptos? ¿Para qué sirven?

El concepto de autorregulación fue introducido por Albert Bandura en el año de 1971 al abordar su teoría de "aprendizaje social”. Según Bandura (1991) entre las variables determinantes del aprendizaje autorregulado se encuentran las que conciernen al ser como individuo -como la motivación, los conocimientos previos o las estrategias de aprendizaje- las referidas a la actuación, o conductuales como la auto-observación, la autoevaluación, la autorreacción y las contextuales, que corresponden al entorno donde se produce la autorregulación o la retroalimentación externa.

Brown (1978, p 77) define a la autorregulación como "la reflexión consciente sobre el propio conocimiento durante el proceso de aprendizaje" precisa que es un proceso que tiene que ver con los manejos de los recursos cognitivos para el control de aprendizajes duraderos y necesarios a lo largo de la vida, mediante la planificación, monitorización y evaluación, estrategias que el individuo emplea para la resolución de problemas (Sáiz y Montero, 2015; Veenman, 2011). En este proceso autorregulatorio, la 
utilización del conocimiento previo es necesario para la evaluación el autoaprendizaje, como también la definición de metas de aprendizaje establecidas por el alumno, que lo llevan a un proceso de reflexión constante sobre lo que quiere lograr y en cuanto tiempo.

Holec (1981) define el proceso de autorregulación como la habilidad que desarrolla un individuo para hacerse cargo de la conducción de su propio aprendizaje, mediante la adquisición de herramientas intelectuales y sociales, que le facilitan la gestión de sus propios aprendizajes y la generación de una autonomía creciente en sus estudios académicos. El desarrollo del proceso de autorregulación, como las metas de aprendizaje y la utilización de conocimientos previos, incorporados en la estructura cognitiva de los individuos, pueden permanecer a lo largo de la vida (Moreno y Martínez, 2007, p. 52), sumándose también la consciencia de los procesos socio-afectivos (Crispin, 2011).

Por último, Moreno y Martínez (2005), subrayan el esfuerzo pedagógico del profesor, necesario para orientar al estudiante a resolver aspectos concretos de su propio aprendizaje durante la revisión de su planificación, control y evaluación de comportamientos, que lo conducirán la realización de una tarea. Este apoyo pedagógico, es necesario hasta que el estudiante muestra avance personal en su autonomía emocional y cognitiva, a través de la observación de su capacidad de decidir por sí mismo, guiado por sus intereses y necesidades personales (Sepúlveda, 2003), agregándose el contexto de su capacidad de trabajo en equipo (Martín, 2008).

La conceptualización anterior da cuenta de que, para vivir en el siglo XXI se tiene como prioridad que los individuos estén prestos a aprender. de acuerdo con el contexto presente y los cambios que se manifiestan, que tenga la capacidad de utilizar conocimientos básicos modificables y uniformes para generar otros. Cobra aquí importancia la frase de Abraham Lincoln no tengo en mucha estima a las personas que no son hoy más listas que ayer, comentario que expone la necesidad de la capacidad de adaptación para vivir nuevas situaciones que el mundo ofrece y transformarse constantemente. Una persona presta a vivir estos cambios, es una persona que ha aprendido a aprender.

De las características enunciadas, podemos afirmar que entonces el aprender a aprender es un proceso, es decir; un conjunto de asociaciones de aprendizajes encadenados al ser humano, que se desarrollan en un tiempo y un espacio que puede ser infinito, cuyas fases conducen a un objetivo específico, mismo que muestra la apropiación de conocimientos y competencias para desplegar su potencial a lo largo de su vida. Entonces, supondríamos que para aprender a aprender, el papel del profesor sigue teniendo una actuación importante y que requiere de una formación y/o actualización pedagógica para adquirir el compromiso para construir, desarrollar y utilizar 
sus habilidades en alumnos que tienen la disposición y motivación para aprender en situaciones contextuales variadas.

\section{METODOLOGÍA}

El presente trabajo está construido bajo la metodología de un estudio detallado, selectivo y crítico que integra la información esencial en una perspectiva unitaria y de conjunto (Icart y canela, 1994). Su finalidad es examinar la bibliografía publicada y situarla en cierta perspectiva (Ramos et al, 2003). La revisión parte de interrogantes que guiaron la recolección de datos, mismos que se analizaron, se establecieron unidades de análisis y se extrajeron conclusiones. Se planteó como objetivo realizar una revisión descriptiva de la literatura que nos permita identificar elementos clave para dar respuestas a nuevas preguntas, así como la identificación a aproximaciones teóricas. Se seleccionaron aquellos documentos que contenían aspectos formales y se hizo una revisión: lectura crítica de documentos, las etapas de realización de una revisión bibliográfica o la elaboración de mapas mentales o mapas conceptuales.

\section{EL APRENDER A APRENDER EN LA ESCUELA SECUNDARIA}

Siguiendo a Delors (1996) en su énfasis en la educación durante toda la vida, que implica la formación de los nuevos ciudadanos para enfrentar las transformaciones de la vida actual en un mundo complejo, y que los contenidos de las materias tienen que fomentar el deseo de aprender, nos interesa mencionar la experiencia de un director de una escuela secundaria con los profesores y estudiantes, subrayando el alejamiento que prevalece entre el ambiente escolar y los requerimientos de actuales de enseñanza y aprendizaje, centrados en el concepto de aprender a aprender.

Para empezar, una de las preguntas que surgen es ¿Cómo el profesor puede armonizar el avance del desarrollo científico y tecnológico, con los planes de estudio y las necesidades del conocimiento de los alumnos? Ellos requieren de una serie de habilidades, como el dominio de las tecnologías de la información y comunicación, así como la investigativa. Al respecto, Tedesco (2011) señala la necesidad de desarrollar la competencia del aprender a aprender del profesor como guía de los educandos, también la de su adaptación para el cambio de actitudes y roles, y expone algunas preguntas ¿Cómo transformarse para ejercer la docencia en este mundo cambiante? ¿Cómo entender la cultura del siglo XXI? ¿Cómo emprender este camino?

La unesco, a través del pensamiento complejo de Edgar Morín (1999) expresa el desafío de la educación del futuro, aprender a aprender de docentes y estudiantes, señalando que ambos enfrentan un paradigma donde se 
definen nuevos métodos y problemáticas, generadas por las diversas formas de vida, mismos que contradicen el enfoque tradicional de los espacios de enseñanza y el aprendizaje. La necesidad de la transformación de las prácticas de enseñanza, han generado crisis al profesorado, algunos porque no cuentan con las herramientas necesarias para hacer frente a estos cambios de paradigma y a otros, porque se resisten a dichos cambios.

Como respuesta a esta visión del aprendizaje, el Sistema Educativo Mexicano, orientado por el pensamiento filosófico expresado en el Artículo $3^{\circ}$, establece que la educación es un derecho de los mexicanos que debe tender al desarrollo armónico de los seres humanos; perspectiva humanista que tiene la finalidad de potenciar los conocimientos, habilidades y competencias que los niños y adolescentes precisan para alcanzar su plena realización (SEP, 2016: 39). Pero ¿Dónde se inscribe la concepción de aprender a aprender para que el profesor adquiera este nuevo hacer?

Por otro lado, en la educación básica, el plan 2011 se presenta como un diseño por competencias, señalando el desarrollo de estas para la vida, es decir competencias para el aprendizaje permanente, que no es otra cosa que la satisfacción de las necesidades actuales, así como la forma de afrontar riesgos permanentes de error y de ilusión, Este marco contempla la habilidad lectora, la habilidad de la escritura, el dominio de más de una lengua, así como habilidades digitales, señalando la competencia de aprender a aprender.

Aprender a aprender aparece aquí como una herramienta más, de la misma magnitud que las que le acompañan como las habilidades digitales o la lectura y la escritura, herramientas necesarias para seguir aprendiendo y con ello la presencia del aprendizaje permanente. Entonces la categoría aprender a aprender no se observa como un espacio diferente que contenga elementos propios, sino que se une a la gama de estrategias para lograr este objetivo. Entonces ¿Qué significa aprender a aprender?

El plan de la educación básica (2016, p. 39) propone que la función de la escuela "ya no es enseñar a los niños y jóvenes lo que no saben, sino aquello que necesitan para aprender a aprender". Es decir es un espacio eminentemente enfocado a las estrategias de aprendizaje, de cómo aprender los contenidos y no de los contenidos en sí mismo. De manera reiterativa enuncia que además de la comprensión lectora y la expresión escrit, agrega el entendimiento del mundo natural y social, razonamiento analítico y crítico, así como la creatividad. Conocimientos y habilidades que se enfatizan como fundamentales para aprender a aprender (SEP, 2016, p.40).

Este plan trae como consecuencia la transformación de la enseñanza y el aprendizaje e implica la construcción de metodologías diversas que apoyen el proceso de formación, reconstrucción y actualización del profesor de su práctica educativa y pedagógica. Esto no ha sido posible, más bien 
existe confusión y confrontación entre los profesores por la falta de claridad de cómo llevar a la práctica educativa el concepto educativo de aprender a aprender.

Sabemos que la escuela de hoy ha dejado de ser el único lugar para aprender, debido entre otras cosas, a la infinidad de fuentes de información, hecho que obliga al profesor a replantear la educación y su práctica educativa. La necesidad de la creación de espacios de apoyo de los profesores que tengan como propósito guiarlos hacia el proceso de aprender a aprender, es fundamental. No se les puede solicitar que enseñen un proceso que ellos mismos no han experimentado. Sumada a esta necesidad, el profesor requiere de herramientas para enfrentar el contexto de violencia al interior y exterior de la escuela. Asimismo, para saber cómo manejar la diversidad, inclusión y equidad como otros tantos problemas que enfrenta en la vida del aula.

\section{ALGUNOS PROBLEMAS DE LA VIDA EN LAS AULAS}

\section{Diversidad, inclusión y equidad}

Una escuela que tenía la tarea de transmitir conocimientos a una población restringida, bajo el principio de que todo el mundo debía aprender lo mismo y al mismo ritmo, se convierte ahora en una escuela que trabaja con la diversidad, con la inclusión y con equidad, lo que transforma la forma de enseñar y aprender. Se suma a esta comprensión del nuevo pensamiento, la influencia de las emociones en el proceso de la actuación profesional de la docencia, donde sabemos que estas dejan una huella duradera, positiva o negativa, en los logros de aprendizaje. Por lo que la función de la escuela tendría que transformarse para generar espacios donde aprender las metodologías adecuadas. Aquí el profesor se incluye emocionalmente, así como los estudiantes, para aprender a pensar de la mano con motivaciones personales. Asimismo, para valorar lo que se aprende en conjunto con los otros, se debe fomentar el interés y la motivación por aprender a lo largo de toda la vida (Delors, 1996).

Hoy se habla del problema metodológico, en el que incluyen factores como: la diversidad, inclusión y equidad, esta es una llamada de atención para decir que el contexto del aula adquiere hoy diferentes connotaciones que implican a la docencia. Los discursos sobre la diversidad han resaltado que la diferencia es un valor, señalando que no se trabaja con alumnos especiales, sino con personas diferentes, por lo tanto la escuela es un espacio de diferentes, porque en la escuela actual convergen estudiantes de distintos contextos y condiciones que conforman una comunidad plural. Particularmente, en la educación secundaria, que ha recibido esta nueva modalidad de trabajo denominada "trabajar con la diferencia". Si los alum- 
nos son diferentes y los profesores son diferentes ¿Cómo amalgamar este binomio de interacción del aprendizaje y la enseñanza?

La escuela tradicional había trabajado de una manera: aprender lo mismo y al mismo ritmo, para iguales, hoy se entiende que la mejora de los aprendizajes tiene que ver con la inclusión, para abordar la diferencia. Además, también se pretende hacer a un lado el memorismo que la tradición conformó en una metodología de aprendizaje y enseñanza, como estrategia básica en la práctica pedagógica. Ahora, la pregunta es ¿̇cómo transformarla o convertirla en herramienta útil para el autoaprendizaje?

La premisa fundamental de que la sociedad está integrada por la diversidad, donde ahora el ámbito de aprendizaje es un derecho universal, independientemente de sus características individuales, por lo que el concepto de inclusión educativa ha evolucionado en los últimos años, ya que suele asociarse con estudiantes que viven situaciones de pobreza o que tienen necesidades especiales. Actualmente se ha logrado un consenso entre los países de América Latina, al ampliar el concepto para lograr un mayor acceso educativo, en busca de una educación de calidad sin discriminación alguna, considerando a los alumnos y alumnas con discapacidad, poblaciones indígenas, poblaciones rurales, migrantes o estudiantes que han abandonado la escuela.

Hacer una aula inclusiva ha sido un reto para los profesores y directivos, porque las diferencias permeaban el contexto, los alumnos más inteligentes, los alumnos más retrasados, los alumnos aprobados, los alumnos reprobados; la decisión del profesor sobre la conducción del grupo, entre otros aspectos que encuadran en el contexto de la práctica docente. Sin importar el desarrollo emocional o cognitivo, los profesores se ven involucrados en una transición de un modelo, en el que su rol era el centro de las decisiones, a otro participativo, en el que el profesor se ha convertido en mediador del proceso de enseñanza y aprendizaje. El tema de discusión aquí es si los procesos de aprendizaje se ven enriquecidos por este modelo, o si los profesores cuentan con las estrategias y herramientas que sirvan de apoyo para desarrollar una educación inclusiva

Lo que sí sabemos es que, en el tema de la inclusión y la equidad, los profesores se ven envueltos en confusiones generadas por la transición que se vive, primordialmente se les pide que los alumnos sean evaluados de acuerdo con su desempeño, donde no existe una metodología que permita la inclusión de todos los alumnos en el trabajo áulico, porque una oferta homogénea de oportunidades reproduce desigualdad en los aprendizajes. Por ello se entiende que es necesaria una oferta con equidad, para aprovechar la riqueza de la diversidad y trascender a una redistribución, reestructuración y cambio de la oferta educativa ¿Cómo hacerlo? No hay claridad sobre el 
apoyo teórico y/o metodológico que pueda facilitar al profesor para atender de manera precisa este propósito educativo.

Como estrategia para intervenir en el cambio en las escuelas, surge el trabajo colegiado de profesores y directivos, organizados en el Consejo Técnico Escolar (сте), órgano colegiado, con mayor decisión, en el ambiente técnico y pedagógico de cada escuela de Educación Básica. Está encargado de tomar y ejecutar decisiones enfocadas a alcanzar el máximo logro de los aprendizajes del alumnado. En la séptima reunión colegiada del total de ocho en el ciclo escolar, se denominó "una escuela inclusiva conoce a sus alumnos". Siguiendo esta guía para el trabajo colegiado, los miembros discutieron sobre la diversidad de alumnos en sus grupos, preguntándose sobre los avances en la inclusión con equidad en la escuela.

En la búsqueda de que los profesores se involucren en esta nueva forma de abordar el aprendizaje, éstos presentaron tres alumnos de cada uno de los grupos que están en riesgo de no alcanzar los aprendizajes esperados o en situación de exclusión, con el objetivo de generar formas de atenderla diversidad desde la participación colegiada. En esta reunión se construyeron las estrategias para atender los problemas de aprendizaje relacionados con las emociones, también para las complicaciones propias que tienen los estudiantes en cada asignatura, como la disposición para aprender, o incluso problemas de comprensión de la materia.

En estas reuniones se reitera la mención de que la problemática de estos alumnos rebasa la capacidad de atención, por ejemplo, hay niños con deficiencia visual y no se atiende el problema, o lo más común, las situaciones emocionales familiares que afectan su rendimiento escolar, donde el profesor no cuenta con las herramientas para apoyarlos. Sumado a lo anterior, el profesor se enfrenta a problemáticas en las que su actuación tropieza con la cultura de los alumnos engendrados en este mundo de transformaciones, la cultura de los profesores y el contraste de la nueva cultura educativa, donde la reprobación y deserción en el modelo de la educación inclusiva, significa etiquetar, discriminar y excluir. Es difícil ahora distinguir si la reprobación es debido a la falta de conocimientos esperados para determinado grado escolar o por causas discriminatorias.

\section{Violencia y delincuencia}

Los contextos externos de la escuela se encuentra influidos por el vandalismo, donde opera la violencia. Ante esta situación, el profesor convive con los ambientes agresivos, sin encontrar respuesta acertada a estas situaciones, que se convierten en contextos de la cultura escolar, así como las limitaciones para responder a los efectos de estas actuaciones, que hacen un campo complejo. Los personajes con características delincuenciales, se convierten en modelos 
de vida de los alumnos, disminuyendo la motivación hacia el proceso de enseñanza y aprendizaje. Animan las acciones de los alumnos con su posición social y poder, justificados por este contexto de vida: no traer los materiales para el trabajo, dificultando con su actuar la conducción grupal; la necesidad de mediar con los líderes para lograr el trabajo en las aulas.

Este tipo de situaciones son solo una de algunas que tiene que sortear el profesor, donde aun viviendo estas circunstancias, se señala la falta de compromiso para el cumplimiento de los objetivos de aprendizaje. Ante esta diversidad de situaciones cabe el cuestionamiento ¿Cómo los modelos educativos, analizan los contextos escolares para emitir su metodología de trabajo?

Frente al contexto del problema de la inclusión y la violencia, se le deja la tarea de aprender a aprender al profesor, con las competencias necesarias para enfrentar estos escenarios, además de cumplir con el fin educativo establecido en el Artículo $3^{\circ}$ constitucional, que se refieren al desarrollo armónico de todas las facultades del ser humano. Además, estar preparado a los cambios de la sociedad del conocimiento, que le exige adquirir mayor capacidad de interpretación de los fenómenos, creatividad y manejo de la información en entornos cambiantes.

La escuela ha dejado de ser el único lugar para aprender, la infinidad de fuentes de información evocan circunstancias que obligan a replantear a la educación obligatoria, donde el desarrollo del pensamiento crítico, el análisis, el razonamiento lógico y la argumentación, solo son parte de un aprendizaje que facilita la resolución de problemas a lo largo de la vida. Mientras que la otra parte es el desarrollo humano y el manejo socio afectivo, que son aspectos fundamentales para comprender y enfrentar los cambios que impactan emocional y cognitivamente al ser humano. El profesor tiene frente a él un problema complejo, representado por un grupo de individuos -los alumnos- que sintetizan el avance del cambio cultural de la sociedad del conocimiento, que progresa mucho más rápido que la cultura educativa de su contexto, de sus herramientas y su saber pedagógico.

\section{APRENDER A APRENDER EN LA EDUCACIÓN SUPERIOR}

En el contexto de la educación superior y, particularmente, en la formación para la investigación, el concepto de aprender a aprender tiene connotaciones como el de desarrollo de procesos cognitivos complejos y el pensamiento flexible, la creatividad, la innovación, la autorregulación, orientados para el desarrollo autogestivo del alumno, idea cada vez más reforzada en las planteamientos educativos que se proponen revisar las necesidades de aprendizaje presentes y futuras de los universitarios. La cuestión es, si en los programas educativos los docentes cuentas con la estructura académica 
para desarrollar estos procesos o si se da por hecho que los estudiantes ya aprendieron a ser autogestivos y no es parte de su función docente.

Esta concepción educativa implicaría sembrar, primero en los profesores, la concepción del significado de un aprendizaje continuo para el desarrollo de la creatividad y la innovación, que son objetivos para el desarrollo de las habilidades investigativas. En cualquier modalidad educativa, el profesor es el que guía, de alguna manera, el aprendizaje del estudiante, por ello la necesidad de la reestructuración de sus esquemas de pensamiento, para la impartición de una clase, la tutoría y la dirección de una tesis.

Particularmente en la formación para la investigación, se menciona la necesidad de una configuración de las capacidades para enfrentar problemas de alta complejidad e incertidumbre, incluso, con la preparación cognitiva suficiente para la generación de insights innovadores que posibiliten el descubrimiento de caminos alternativos para la comprensión de aquellos problemas que tienen una estructura difícil de definir (Abreu y De la Cruz, 2015). Es un avance el empezar a discutir este perfil de estudiantes que se relaciona con el desarrollo de la creatividad, expertos en la formación para la investigación así lo confirman (Moreno, 2013). El problema es que la comprensión del significado conceptual no es suficiente para saber qué herramientas cognitivas y pedagógicas son necesarias para preparar a los estudiantes a enfrentar la incertidumbre y, sobre todo, conducirlos hacia un insigth.

Si bien estos procesos son importantes en los estudios de posgrado, en la formación para la investigación toman mayor relevancia para facilitar la apertura cognitiva en el planteamiento de problemas de investigación pertinentes a las necesidades sociales. El desarrollo de la flexibilidad de pensamiento, la creatividad, el pensamiento complejo y tolerancia a la incertidumbre, representan procesos complejos de comprensión en sí mismos. Tanto como el conocimiento de las prácticas pedagógicas adecuadas para los estudiantes y metas educativas e investigativas.

Es escasa la investigación sobre las herramientas pedagógicas que puedan orientar a los estudiantes hacia la apropiación de las herramientas cognitivas para la comprensión de los aprendizajes personales. Al respecto, parece que se ha puesto demasiado énfasis en la necesidad de resolver problemas complejos y menos atención a las dificultades cognitivas que pueden impedir la percepción y el afrontamiento de dicha complejidad, tanto del docente como de los estudiantes.

La revisión de estas dificultades, por parte del profesor, implican entre otras muchas tareas, la recapitulación de su práctica educativa, la recuperación y reestructuración de su experiencia y conocimientos para integrarlos a su propio aprendizaje, para encarar los problemas que la sociedad actual demanda fuera y dentro del contexto escolarizado. También se deben re- 
pensar las prácticas educativas o pedagógicas que se complementen con los recursos tecnológicos para interactuar con los estudiantes en la gestión del conocimiento. Es en esta compleja situación donde surgen cuestionamientos que se plantean en la educación superior, entre ellos, la reinvención del concepto mismo de aprendizaje en tanto proceso continuo, la creación de herramientas cognitivas que requieren profesores y estudiantes para entender y hacer frente a una realidad compleja, así como qué propuestas tecnológicas, para el desarrollo de la creatividad.

Como es sabido, la mayoría de los docentes universitarios y de posgrado no tienen una formación pedagógica, su práctica educativa es producto de la experiencia y modelos aprendidos durante su formación profesional. Por tanto, al igual que en la educación básica puede haber confusión en algunos conceptos que involucran el aprender a aprender en este nivel educativo, como la regulación y autogestión del aprendizaje, la tolerancia a la ambigüedad, el significado de un insigth, etc., así como también de las prácticas pedagógicas que pueden favorecer estos procesos.

En el caso de la formación para la investigación, las prácticas pedagógicas, en muchos casos, no son orientadas hacia este propósito, sino a loa tradicionales contenidos curriculares, de poco interés para los estudiantes, cargas abundantes de lecturas en los seminarios que, aunque tienen intenciones formativas para la investigación, no siempre se cumplen. Para atender esta situación, se sugiere mejorar la calidad de la instrucción, que implica la organización de las experiencias de aprendizaje a vivir, fuera y dentro del aula (Moreno, 2006). También se debe orientar la perspectiva de la dirección de tesis como una práctica pedagógica para la valoración de estrategias efectivas para el aprendizaje de la investigación (Fernández y Wainerman, 2013). Es insuficiente la investigación sobre las estrategias pedagógicas para desarrollar en el estudiante herramientas cognitivas que favorezcan el aprendizaje continuo. Como ejemplo de algunas de ellas, expondremos tres propuestas para desarrollar algunos procesos cognitivos relacionados con el aprender a aprender; la primera, trata sobre el beneficio de saber elaborar preguntas indagatorias, la segunda expone la implicación reflexiva de la elaboración de mapas conceptuales y la tercera describe la importancia de las metas de aprendizaje.

\section{Saber elaborar preguntar indagatorias}

Un tema recurrente en las propuestas para la formación de los estudiantes universitarios y, sobre todo, en la formación de investigadores, es que los estudiantes cuenten con las herramientas para enfrentar la complejidad de los problemas actuales. Dichas herramientas cognitivas se deben concebir como una postura ante los problemas educativos, para desarrollar 
la creatividad y la autogestión, así como utilizar la información de manera educativa (Organista, Serrano, MacAnally y Lavigne, 2013). Sin embargo, cabe la pregunta de si en los contextos educativos se les brindan dichas herramientas, como por ejemplo, aprender a cuestionar la realidad. ¿Es posible enfrentar la complejidad sin cuestionar? Elaborar preguntas representa un ejercicio cognitivo para la comprensión de uno mismo, de los otros y del contexto social.

Para desarrollar la creatividad es necesario incentivar la curiosidad en los estudiantes, y para ello, es necesario enseñarlos a preguntarse sobre sus intereses, motivaciones y problemática académica. Un ejemplo del valor pedagógico de la pregunta en el aula de clases para desarrollar la observación indagatoria de la realidad la expone Plata (2011) como una opción en la formación de estudiantes universitarios con el propósito de aportar a una educación para la incertidumbre. Fundamentándose en las ideas de Gadamer y Freire, habla sobre los beneficios de utilizar la pregunta como herramienta para que el estudiante aprenda a pensar por sí mismos, sirva de guía para introducirse al conocimiento reconociendo sus intereses personales y formular hipótesis explicativas desde su propia experiencia. Retomando a Gadamer (1999), plantea que la base del deseo de saber "presupone un saber que no se sabe, siendo la determinación de no saber lo que da ese primer sentido de apertura a la pregunta; esa es la razón por la que todo saber debe pasar primero por la pregunta" (p.148). En esta experiencia de investigación, se muestra la importancia de hacer preguntas es desconocida por los estudiantes, incluso, no es apreciada como una tarea vinculada a la labor de investigación, ni tampoco para su actividad profesional. La respuesta que exponemos, de uno de los participantes, nos da una evidencia para reflexionar acerca de cómo en la labor docente podemos dar respuestas sin preguntas o conocimientos digeridos sin relacionarlos con los intereses personales de los estudiantes. Para Organista (et al, 2013) al interrogar sobre qué idea tienen, responden "nos acostumbramos a que todo nos lo dan entonces no sabemos reaccionar con preguntas, comunicarnos con preguntas para desarrollar más intereses y más conocimientos. Entonces se corta el espíritu investigador".

Esta expresión sobre "todo se nos da" no solamente puede aludir a los docentes, sino a la información que obtienen a través de diferentes dispositivos tecnológicos, que son accesibles a la mayoría de los estudiante, que no obstante, se ha observado que no son utilizados como una herramienta para el beneficio educativo personal (Plata, 2011,p. 18). Las preguntas indagatorias pueden ser generadas también por el propio estudiante en el transcurso de la exposición del docente en el aula de clases. Se ha observado en las investigaciones sobre la utilidad de la los apuntes y anotaciones para el aprendizaje, los estudiantes que aplican las estrategias del resumen y 
además, las estrategias de autointerrogación mejoran la comprensión como el recuerdo de la información (Espino y Miras, 2013).

Consideramos que esta práctica de hacer preguntas en las notas de clase, ha sido una experiencia pedagógica vívida. Recientemente, en un curso de posgrado, se les solicito a los estudiantes entregar un reporte escrito a mano sobre las lecturas sugeridas; no se les pide un resumen, sino las reflexiones personales que las lecturas les provocaron. Dos de ellos entregan su reporte y le añaden interrogantes personales, relacionados con las dudas con las posturas de las teorías revisadas y también vinculadas con su tema de investigación de tesis. Interrogantes que se toman como parte de los temas a discutir en clase, con la intención de cubrir los espacios vacíos de aprendizaje no solo de este estudiante sino también de los otros. De esta manera las preguntas fungieron como el lazo vinculante de la clase anterior con la presente.

Implicación reflexiva de los mapas conceptuales

En la educación superior, la necesidad de actualización docente para la revisión de su práctica pedagógica en la elaboración de materiales y herramientas, propias a su estilo personal y materias a impartir, es relevante. Con el desarrollo tecnológico se han ofrecido varias alternativas para el docente, la incorporación de juegos como estrategia de aprendizaje, materiales educativo multimedia, entrenamientos desde la plataforma etc., Moodle (Díaz, Villalobos González-Pienda y Nuñez, 2017). En esta última investigación, los autores resaltan el papel del profesor, tanto en el aprendizaje virtual como el presencial, mencionan que el profesor representa un referente para apoyar a los estudiantes en un aspecto importante para el desarrollo de la autonomía del estudiante hacerle ver al estudiante las capacidades que éste no percibe de sí mismo.

Desde esta idea, las investigaciones que exploran el entrenamiento de habilidades pedagógicas en la formación docente son relevantes porque pueden ser incorporadas previamente a su práctica profesional. Asimismo, dicho entrenamiento puede revelarles sus capacidades e incapacidades, que lo posicionan en la experimentación del proceso de aprendizaje tal como un estudiante lo vive. Reyes y Ramos (2018) nos presentan una investigación con profesores de séptimo semestre de la carrera de pedagogía, que se propone observar el beneficio de elaborar mapas conceptuales para su utilización como herramienta para el aprendizaje y también como estrategia que posibilita un proceso metacognitivo.

Los autores muestran cómo al elaborar los mapas conceptuales sobre las nociones de didáctica, matemática y diseño de clases, así como también los motivos que tuvieron para hacerlos de esa manera, sucede una progresión, tanto del aprendizaje como del tratamiento de elaboración conceptual. Se resalta 
la dificultad de los participantes para la elaboración de los mapas conceptuales; durante el proceso de la realización de la tarea, van descubriendo los problemas cognitivos y emocionales que implican la reestructuración de su pensamiento, la selección de los conocimientos, la organización de ideas y la jerarquización de conceptos. El Proceso reflexivo es constante, por lo que a la vez que se observa la inducción hacia la metacognición, se subraya la progresión del pensamiento del profesor, evolucionando en el sentido de reconocer sus problemas para sintetizar su conocimiento, ordenarlo conceptualmente, etcétera.

La relevancia de esta investigación es la implicación reflexiva del profesor, mediante el aprendizaje de una herramienta que le facilite desarrollar su pensamiento, rompiendo estructuras fijas de cómo presentar el conocimiento. Asimismo, este proceso, que lleva a la metacognición, experimenta el proceso de aprender que puede transferir posteriormente con sus estudiantes.

\section{Metas de aprendizaje y evaluación formativa}

El diseño de las metas de aprendizaje, centradas en el desarrollo de la competencia y el dominio de la tarea, representa uno de los componentes del proceso de la autorregulación del aprendizaje y ha sido tratado en las investigaciones actuales, tanto en sus postulados teóricos como intervenciones pedagógicas (Valle, Rodríguez, Núñez, González-Pienda y Rosario, 2007). La elaboración de metas es una actividad compleja, que involucra un reordenamiento cognitivo para jerarquizar intereses y reconocer las motivaciones para llevarlas a su realización. Desde la teoría de los estilos de pensamiento de Sternberg (1999), esta actividad cognitiva se representa en el pensamiento jerárquico, manifestado en la planificación de sus procesos mentales, en función de sus metas personales, académicas o no académicas, además es un pensamiento que potencia el desarrollo de la creatividad.

Algunos trabajos empíricos han mostrado que el pensamiento jerárquico contribuye a desarrollar la consciencia metacognitiva (Valadez y Moreno, 2017). Al respecto, se realiza una intervención pedagógica con estudiantes de posgrado, para observar si el reconocimiento de su estilo de pensamiento puede ser una herramienta metacognitiva que le facilite detectar problemas en su avance de investigación de tesis. Los resultados muestran que el escaso desarrollo del pensamiento jerárquico afecta negativamente para la decisión del tema de investigación (Valadez, 2016). Tomando en cuenta estos resultados, se plantea que el reconocimiento de los estilos de pensamiento puede iniciar un proceso autogestivo en los estudiantes, particularmente, el desarrollo del pensamiento jerárquico, que puede ejercitar la definición de metas orientadas a los intereses personales. 
La relación entre las metas de aprendizaje y la evaluación, se centra en considerar un proceso de evaluación formativa en el sentido de que ésta última se convierte en una oportunidad para apoyar el aprendizaje a partir de promover la autorregulación. Este tipo de evaluación fomenta que el estudiante decida sus propias metas de aprendizaje, tomando en cuenta su contexto y necesidades personales, incluso su propio estilo de pensar, con el propósito de que disfrute su aprendizaje. Así, la evaluación es una recapitulación sobre el cumplimiento dicha meta, actividad que favorece además la metacognición y el reconocimiento del error como parte de su aprendizaje (Álvarez, 2008).

\section{CONCLUSIONES}

Como se mencionó a lo largo de este trabajo, el concepto de aprender representa un concepto deseable, más no sostenido, por la práctica educativa. Si bien la educación básica y la superior tienen distintos referentes, lo que prevalece en ambos son las prácticas tradicionales y los entornos educativos que no favorecen el cambio de percepción de la enseñanza. La complejidad social, representada en los alumnos en las aulas de clases de educación básica, ha rebasado al docente y a la estructura escolar. Se requiere investigación educativa que incluya el contexto para conocer las características propias de la escuela y brindar alternativas y apoyo pedagógico a los docentes para iniciar un cambio de percepción del aprendizaje.

Sería deseable que el profesor, tanto del nivel básico como del superior, experimente el cambio del pensamiento para aprender a aprender, si esto no sucede, es difícil que pueda transferirlo a sus alumnos y la distancia entre el concepto y la práctica pedagógica estará presente, sobre todo en la educación superior, y los posgrados, que pretenden el desarrollo autogestivo de los estudiantes para la innovación y la creatividad, mismos que requieren cambios en la práctica educativa.

Las propuestas pedagógicas presentadas, dan cuenta de la importancia del diseño pedagógico de una clase para enseñar a pensar; también de que la actividad de la lectura no es suficiente para su comprensión, aún en cantidades abundantes. Es necesario utilizar estrategias, como un andamiaje, para que los estudiantes construyan su aprendizaje y elaboren sus propias herramientas cognitivas.

Aprender a aprender implica esfuerzo, disposición e interés del profesor y el estudiante, por la actividad reflexiva permanente, no solamente sobre el conocimiento sino sobre sí mismo y la relación con los otros. Es una tarea y tema de investigación conocer la distancia entre este concepto y la vida cotidiana del ámbito educativo y personal. 


\section{REFERENCIAS BIBLIOGRÁFICAS}

Alvarez, I. M (2008). Evaluar para contribuir a la autorregulación del aprendizaje. Electronic Journal of Research in Educational Psychology $7(3), 1696-2095$.

Abreu, L. F., y De la Cruz, G. (2015). Crisis en la calidad del posgrado ¿Evaluación de la obviedad, o evaluación de procesos para impulsar la innovación en la sociedad del conocimiento? Perfiles educativos, 37(147), 162-182.

Bandura, A. (1971). Analysis of modeling processes. In A. Bandura (Ed.), Psychological modeling: Conflicting theories. Chicago: Aldine-Atherton.

Brown, A. L. (1978). Knowing when, where, and how to remember: A problem of metacognition. En R. Glaser (Ed.), Advances in Instructional Psychology (Vol.1, pp.77-165). New York: Halsted Press.

Brunner, J. (1998). Globalización Cultural y Posmodernidad. Chile: FCE.

Brunner, J. y Tedesco, C. (2003). Las nuevas tecnologías y el futuro de la educación. Buenos Aires. IIPE Unesco.

Crispín, B. (2011).Aprendizaje autónomo: orientaciones para la docencia. México: Universidad Iberoamericana.

Departamento de Educación del Gobierno Vasco. (2012). Competencia para Aprender a Aprender. Recuperado desde: http://ediagnostikoak. net/edweb/ cas/materiales-informativos/ ED_marko_teorikoak/ Aprender_a_aprender.pdf

Delors, J. (1996). La educación encierra un tesoro. París: Santillana-UnEsco.

Diaz, A., Villalobos, M.V., González-Pienda, J.A. y Nuñez (2017). Impacto de un entrenamiento en aprendizaje autorregulado en estudiantes universitarios. Perfiles educativos, 39(157), 87-104.

Escotet, M. (1992). Aprender para el futuro. Madrid: Alianza

Espino, S., Y Miras, M. (2013). El proceso de anotación y uso posterior de los apuntes para el aprendizaje. Revista Mexicana de Investigación Educativa, 18(159), 1257-1280.

Fernández, L. y Wainerman, C. (2013).La dirección de tesis de posgrado: ¿una práctica pedagógica? Perfiles educativos, 37(148), 156-171.

Ferrater- Mora, J. (2009). Diccionario de filosofía. Barcelona: Editorial Ariel.

Gadamer, Hans-Georg (1999), Verdad y método. Salamanca, Ediciones Sígueme.

Holec, H. (1981). Autonomy in Foreign Language Learning: Oxford University Press.

Icart Isern, M. T., \& Canela Soler, J. (1994). El artículo de revisión. Enfermería clínica. 4(4), 180- 184.

Martín, E. (2008). Aprender a aprender: una competencia básica entre las básicas. Madrid: Universidad autónoma de Madrid.

Mayor J. (1995). Aprender a aprender y aprender a pensar. Madrid, Editorial Síntesis. 
Moreno, R.; Martínez, R. (2007). Aprendizaje autónomo. Desarrollo de una definición Acta Comportamentalia: Revista Latina de Análisis de Comportamiento, vol. 15, núm. 1, pp. 51- 62 Universidad Veracruzana. Veracruz, México.

Moreno, M.G. (2006). Formación para la investigación. Guadalajara, México: Universidad de Guadalajara.

Moreno, M. G. (2013). Retos actuales de la formación de investigadores en educación en México. En M. G. Moreno y M. Valadez, Miradas analíticas sobre la educación superior. Guadalajara, México: Universidad de Guadalajara, 415-424.

Organista- Sandoval, J. Serrano-Santoyo, A. MacAnall, L. y Lavigne (2013). Apropiación y usos educativos del celular por estudiantes docentes universitarios. Revista electrónica de Investigación Educativa 15(3) ,138-156.

Morín, E. (2000). Los siete saberes necesarios para la educación del futuro. UNESCO - IESALC, Ediciones Faces/UCV. Caracas

Plata, M. E. (2011). Procesos de indagación a partir de la pregunta. Una experiencia de formación en investigación. Praxis \& Saber 2(3) 139-171.

Ramos, M. H., Ramos, M. F., \& Romero, E. (2003). Cómo escribir un artículo de revisión. Revista de postgrado de la VIa Catedra de Medicina (126), Disponible en http://med.unne.edu.ar/revista/revista126/como_esc_ articulo.htm. [Consultado ocho de octubre de 2019].

Reyes- Santander, P.A., y Ramos-Rodriguez, E. (2018). Mapas Conceptuales en la educación matemática en nivel universitario. Revista electrónica de Investigación Educativa 20(2), 25-36.

Reimers, F. (2016). Teaching and Learning for the Twenty First Century. Cambridge: Harvard Education Press.

Sternberg, R.J. (1999). Estilos de Pensamiento. Barcelona, España.

Sáiz, M.C. \& Montero, E. (2015). Metacognition, Self-regulation and Assessment in ProblemSolving Processes at University. En A. PeñaAyala (Ed.), Metacognition: Fundaments, Applications, and Trends (pp.1-27). doi 10.1007/978-3-319- 11062-2-5.

SEP (2016). Educar para la libertad y la creatividad. México: SEP.

SEP (2016).Modelo educativo 2016. Mexico: SEP.

Sepúlveda R. (2003). Autonomía moral: Una posibilidad para el desarrollo humano desde la ética de la responsabilidad solidaria. Revista de Psicología, vol. 12, núm. 1, 2003, pp. 27-35 Universidad de Chile Santiago, Chile.

Tedesco, J. (2011). Los desafíos de la educación básica en el siglo XXI. Revista Iberoamericana de Educación, 55(1), 31-47. Recuperado de http://rieoei.org/rie_contenedor.php?numero=rie55

Valadez, M. (2016). Indicios para gestionar el aprendizaje personal identificando los estilos de pensamiento. En M. Valadez, M. G. Moreno y C.E. 
Vivero, Avances y perspectivas de la investigación educativa y de género. Guadalajara, México: Universidad de Guadalajara, 107-117.

Valadez, M.; Moreno, M.G. y Romero, M.A. (2017). Transiciones en la formación de posgrado. Recuperación de la experiencia y acercamiento conceptual desde tres enfoques. En V. Ortiz y A. Ponce, Diversas miradas sobre el posgrado y la investigación educativa en Latinoamérica. Guadalajara, México: Universidad de Guadalajara.

Valle, A., Rodríguez,.S. Nuñez, J.C., González-Pienda, J.A. y Rosario, P. (2007) Metas académicas y estrategias de aprendizaje en estudiantes univesitarios. Revista Semestral du Associação Brasileira de Psicología Escolar 11(1),31-40.

Veenman, M. (2011). Learning to Self-Monitor and Self-Regulate. En R. E. Mayer y P. A. Alexander (Eds.), Handbook of Research on Learning and Instruction (pp. 197-218). New York and London: Routledge.

UNESCO (2017). Replantear la Educación ¿Hacia un bien común mundial? Paris. UnESCO. 